\title{
Choosing the Right Catheter for Pediatric Procedures: Patient Considerations and Preference
}

\author{
Chad Crigger (10) \\ Jake Kuzbel (D) \\ Osama Al-Omar (it) \\ Department of Urology, Division of \\ Pediatric Urology, West Virginia \\ University, Morgantown, WV, \\ 26506, USA
}

\begin{abstract}
Determining the need for bladder decompression and urinary diversion in the perioperative pediatric surgical patient can cause a clinical conundrum for the surgical team. Add in the several different types of urinary diversion devices possible, and the various materials associated therein and the process can suddenly seem unnecessarily daunting given the lack of concise recommendations and broad consensus. The decision to divert urine, though seemingly trivial, is associated with inherent risks. Managing and mitigating certain risks are best approached through proper education, selection, and technique. We provide a broad overview of pediatric catheter selection, indications, and pitfalls to streamline the process so that energy and attention can best be focused on the planned intervention at hand. Keywords: pediatric surgery, pediatric urology, urinary diversion, quality improvement, CAUTI
\end{abstract}

\section{Introduction}

An average of 450,000 pediatric patients are admitted each year in the United States for surgery. Elective surgery admissions generally comprise $40 \%$ of the surgical hospitalizations with $55 \%$ of admissions classified as either gastrointestinal, orthopedic, or urological. ${ }^{1}$ Consider the high volume of additional outpatient surgical procedures, representing over $60 \%$ of all surgeries in the United States in 2011 , and the diligence to perioperative management in the pediatric patient is vital. ${ }^{2}$ While the immediate pathology at hand and surgical technique dictated are the primary focus of the surgical team, one facet that receives considerably less attention, if any, is the role of urinary catheter placement.

The decision to place a urinary catheter, or not, or another variant to decompress the bladder may seem trivial. However, a dive into the topic only further complicates the matter. Consider that catheter-associated urinary tract infections (CAUTIs) are the most common nosocomial infection in the United States, accounting for more than $30 \%$ of infections reported by acute care hospitals. ${ }^{3}$ As this is associated with increased healthcare costs, hospital stays, morbidity and mortality rates, there has been a drive to determine when catheters should be utilized. ${ }^{4}$ In fact, national quality improvement measures to strictly determine safe catheter usage are seen in campaigns such as "Lose the Tube" of Choosing Wisely Canada."

When the same dilemma is applied to the pediatric patient, the water is further muddied. Pediatric patients, in fact, are not just small adults. With incompletely developed and developing physiology, more delicate anatomy, in addition to the role urinary catheterization has historically played in the postoperative healing in
Correspondence: Osama Al-Omar Department of Urology, Division of Pediatric Urology, West Virginia University, Suite 1400, Health Sciences Center South, Morgantown, WV, 26506, USA

Email osalomar@hsc.wvu.edu 
certain procedures, selecting the proper catheter - and knowing when to use one - is no simple task.

In what follows, we hope to provide a basis for when catheterization should be employed in the pediatric patient generally. Building on this, we will explore how the intended procedure, approach, and underlying pathology may all influence this selection process so that clinicians may better arrive at a sound decision.

\section{When Should a Catheter Be Used?}

In 2018 Meddings and colleagues set out to determine criteria for urinary catheter use in common general and orthopaedic surgeries using the RAND/UCLA appropriateness method. ${ }^{6} \mathrm{~A}$ bold undertaking, the panel, consisting of two multidisciplinary sub-panels totaling 24 members utilized a standard process to independently rate the clinical appropriateness for Foley catheter placement in 91 general surgical procedures and 36 orthopaedic surgeries. With the aim to limit unnecessary catheterization and prioritize removal when urinary drainage was needed, prompted three recommended categories: (a) procedures for which indwelling urinary catheter placement should be avoided, (b) procedures to consider removing indwelling urinary catheters before leaving the operating room, and (c) procedures in which urinary catheters should remain in use until postoperative day 1 (Figure 1). One obvious omission in this panel review was the lack of review of urologic procedures.

In the general surgical realm, Foley catheter use was highly appropriate for all colorectal surgeries with appropriate timing of first voiding trial occurring as early as postoperative day 0 or postoperative day 1 . Routine catheter placement was deemed inappropriate for several procedures, including laparoscopic cholecystectomy, open appendectomy, laparoscopic appendectomy without a suprapubic port, open repair of reducible hernias (inguinal, femoral, umbilical, epigastric), and most laparoscopic repairs provided the patient voided preoperatively.

One area where catheter placement was most highly dependent upon surgeon experience and training included whether or not suprapubic port placement in laparoscopic surgery impacted urinary drainage strategies. The panel discussed catheter placement may be avoided if the patient voided preoperatively with the option of bladder scanning, but consensus appropriateness remained undecided regarding routine placement. As expected, many practices are determined by surgeon training and if any surgeon had experienced bladder or other urologic complications with suprapubic port placement. By convention, most urologists place urinary catheters, at least intraoperatively, for most pelvic surgeries, including both open and laparoscopic approaches to minimize risk of inadvertent iatrogenic damage to the bladder.

For orthopaedic procedures, the competing interests were the need to decrease catheterization where infections are a real risk to hardware and operative time. In general terms, most routine hip and knee procedures can safely be performed without catheter placement if completed in under two hours. In those that last longer than two hours, catheters could often be removed before leaving the operative suite or on postoperative day 1 with no increase risk in postoperative complications.

\section{Postoperative Urinary Retention - What Is It and What Mitigation Strategies Exist?}

The overall risk of postoperative urinary retention (POUR) in the general (non-urologic) surgical population is $3.8 \%$, though the cited incidence can vary widely from 5 to $70 \%$ based on the type of surgery. ${ }^{7}$ In pediatric patients undergoing lower extremity orthopedic limb surgery, up to onethird of patients may develop POUR. ${ }^{8}$

The alterations in physiology in the postoperative period can result from various types of anesthesia employed. The effects may be due to anesthesia itself, the procedure performed and technique utilized, polypharmacy intraoperatively, and postoperative pain. Although there has been a recent push away from postoperative opiate pain management, opioids are commonly still used intraoperatively and are known to decrease the sensation of bladder filling by parasympathetic inhibition. ${ }^{9}$ Additionally, they increase sphincter tone. The effect, and subsequent urinary retention, is greater with neuraxial opioids compared to intravenous administration. General anesthesia also contributes to urinary retention by increasing smooth muscle relaxation, and, conversely, decreasing bladder contractility. Finally, local neuraxial local anesthetics act at the immediate afferent and efferent junctions in the pathways pivotal to micturition. As such, longer acting agents carry a greater risk for postoperative bladder dysfunction.

While several distinct and competing criteria have been used to diagnose POUR generally the condition can be diagnosed based on history and physical examination, need for bladder decompression, and, more recently, ultrasonography. ${ }^{10,11}$ Suprapubic pain at rest and with at palpation as well as a distended, palpable bladder can 
A. Avoid placing indwelling urinary catheters for these routine procedures: these are procedures for which it is considered inappropriate to place a catheter for the procedure, as the catheter risk is considered to outweigh the benefits for the patient. $\dagger \neq$

\section{General surgery}

- Laparoscopic cholecystectomy

Open appendectomy

- Laparcscopic appendectomy without suprapubic port

- Open reducible inguinal, femoral, umbilical or epigastric hernia repair

- Laparcscopic reducible inguinal or femoral hernia repair by TAPP if bladder emptied before surgery

- Laparoscopic reducible umbilical or epigastric hernia repair

- Laparoscopic adjustable gastric banding

B. Procedures to consider removing indwelling urinary catheter before leaving the $O R$

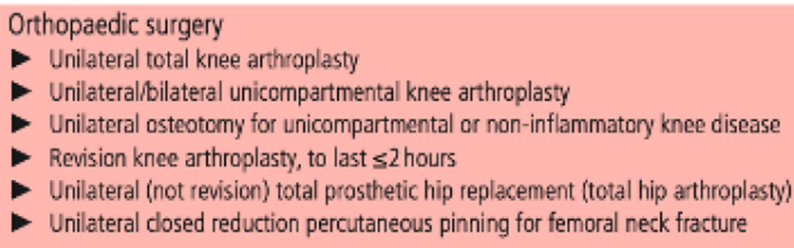

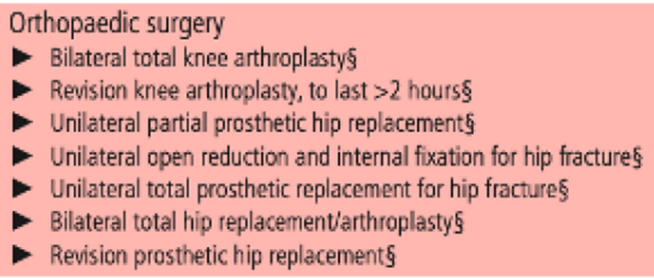

C. Procedures in which urinary catheter use in the OR and until at least postoperative day 1 is appropriate, with the timing for the first trial of void detailed below by procedure

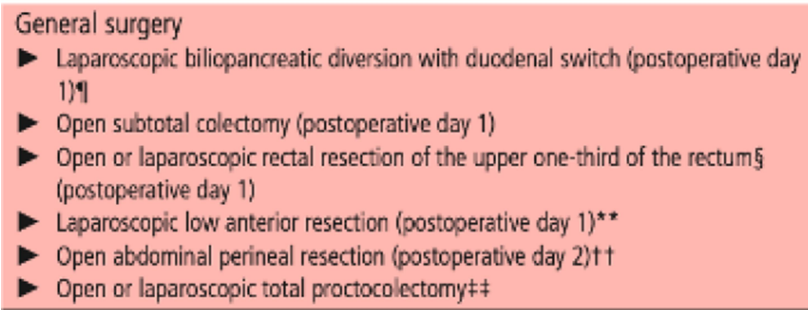
address a medical indication such as critical illness for which hourly urine output is being used to guide therapy such as vasopressors). For all procedures, using a postoperative protocol to monitor and address urinary retention symptoms is recommended; bladder scanners are increasingly common tools to verify retention in patients with symptoms to avoid unnecessary catheterisations.

tRoutine urinary catheter use is not appropriate for these procedures when less than 2 hours of OR time and less than $2 \mathrm{~L}$ of intravenous fluids anticipated in the OR. Experts indicated that routine catheter use during the OR case could be appropriate for procedures $>3$ hours in duration or with $>3 \mathrm{~L}$ of intraoperative fluids.

¥Patients are recommended to woid before surgery. If concemed about postvoid residual, use of bladder scanner protocol with intermittent straight catheter as needed before surgery is an appropriate alternative to routine indwelling catheter use in patients with urinary retention.

§For these procedures, it was assessed also as clinically appropriate to remove catheter on postoperative day 1 .

IFor this procedure, there was uncertainty about appropriateness of routinely removing on the same day of surgery; therefore, it could be clinically appropriate to remove earlier than postoperative day 1 by surgeon's discretion.

${ }^{* \star}$ For open low anterior resection, removal before postoperative day 3 is appropriate, but there was uncertainty for whether removal was more appropriate on postoperative day 1 compared to postoperative day 2 .

t†For laparoscopic abdominal perineal resection, removal by postoperative day 4 is appropriate, but there was uncertainty for whether a particular day within the range of postoperative days 1-4 was more appropriate than others.

¥ \#For open or laparoscopic total proctocolectomy with or without ileal pouch anal anastamosis, removal by postoperative day 4 is appropriate, but there was uncertainty for whether a particular day within the range of postoperative days 1-4 was more appropriate than others.

$\mathrm{OR}$, operating room; TAPP, transabdominal preperitoneal; TEP, totally extraperitoneal.

Figure I Summary of perioperative urinary catheter use recommendations.

Notes: Adapted from Meddings J, Skolarus TA, Fowler KE, et al. Michigan Appropriate Perioperative (MAP) criteria for urinary catheter use in common general and orthopaedic surgeries: results obtained using the RAND/UCLA Appropriateness Method. BMJ Quality \& Safety. 20I8;28(I):56-66. (C Author(s) (or their employer(s)) 20I9. Re-use permitted under CC BY-NC. Published by BMC Author(s) (or their employer(s)) 2019. Re-use permitted under CC BY-NC. No commercial re-use. See rights and permissions. Published by BMJ. With permission from BMJ Publishing Group Ltd. ${ }^{6}$

clue the clinician into ongoing POUR, though this method does lack sensitivity. In adult patients, palpable dullness to the level of the umbilicus is a reliable surrogate in measuring bladder volumes of approximately 500 mL. $^{12}$ Bladder catheterization offers both a diagnostic tool and therapeutic measure for POUR, as some criteria diagnose POUR if $100 \%$ of expected adult volume is drained $(500-600 \mathrm{~mL}$ in adults) and more than the calculated bladder capacity at a certain age in a child $([$ age in years +2$] \times 30) .{ }^{13}$ Finally, ultrasonographic investigation has proven a useful adjunct in diagnosing POUR as it is non-invasive, painless and has a high 
sensitivity, with strong correlation between volumes measured by bladder catheterization and ultrasound. ${ }^{14,15}$

Strategies to decrease the risk of POUR have been heavily studied and resulted in alterations in operative management by the surgical team and anesthesia management. Judicious intravenous fluid administration based on calculated maintenance fluids, and accounting for insensible loss, is imperative. This approach should be employed when possible, though there are instances when a surgeon may request hyperhydration, such as in urologic upper tract reconstruction, to induce a physiologic hydronephrosis.

Other approaches to decrease risk include being cognizant of surgical duration. Keita et al demonstrated that surgical duration alone, specifically procedures lasting 80 minutes or longer, were independently associated with increased risk of POUR. This is likely multifactorial, due to the increased intravenous fluid administration and use of opiate pain control for general anesthesia. However, pain control in the postoperative setting is crucial as poor pain control can result in increased sympathetic discharge which further predisposes a patient to POUR. ${ }^{16}$

\section{Catheter Size in Children - One Size Does Not Fit All}

Catheterization in the pediatric patient, unlike the adult patient, is often performed in conjunction with diagnostic as well as therapeutic procedures. Examples in which catheterization is diagnostic include contrast-enhanced imaging modalities such as that seen in cystourethrography. Though the procedure is commonly performed and relatively safe, there are relative contraindications to immediate catheterization including pelvic fractures, known trauma to the urethra, or blood at the meatus. ${ }^{17}$ Complications are rare considering how frequently catheterization is performed, however, they do arise. Examples include false passage creation, urethral perforation, hematuria secondary to traumatic placement, infection and subsequent delayed complication of urethral stricture formation.

Key to avoiding, or at least minimizing, complications is appropriate catheter size selection. The method for doing so varies widely and traditional selection processes rely on the child's age, body weight, or both. ${ }^{18-21}$ Exact formulas have even been derived to provide an ideal and custom approach to proper catheter sizing in children. One relies on a patient's age to estimate body weight (in $\mathrm{kg}$ ) up to 10 -years: ${ }^{22}$ Weight may not always be the most accurate, however, as there can be great variance of expected weight for a given age. To address this, Kopac devised a formula based entirely on body weight (up to $30 \mathrm{~kg}$ ) and correlated this with catheter size:

$$
\begin{gathered}
\text { Body weight }(\mathrm{kg})=2 \mathrm{x}(\operatorname{age}[\mathrm{yr}]+4) \\
\text { Urinary Catheter }(\mathrm{Fr})=\frac{\text { Body weight }(\mathrm{kg})}{3}+4
\end{gathered}
$$

This formula was verified to help those clinicians quickly determine the appropriate catheter size who may not have readily available access to published nomograms for height and weight or who may not often treat pediatric populations. As the formula is based on published relations between urinary catheter size and a child's age and body weight, the formula holds strong correlation up to $30 \mathrm{~kg}{ }^{23}$

\section{Special Considerations Hypospadias Repair}

Management of urine postoperatively following surgical repair of hypospadias is, in itself, a highly debated topic in the realm of pediatric urology. Classic reasons for placing a urethral stent or other urinary drainage device such as a Foley include the belief that voiding postoperatively may prove painful or cause discomfort that may predispose a child to develop urinary retention - by leaving a urethral stent, the risk for re-cannulating a fresh hypospadias repair is avoided. Additionally, others believe urethral stenting provides a physical framework around which a repair may heal, particularly if the urethral plate is reconstructed and tubularized as in the tubularized incised plate repair (TIP procedure). ${ }^{24}$ To address this exact question, El-Sherbiny prospectively randomized boys undergoing a TIP procedure to either stented $(n=35)$ or unstented $(n=29)$ groups for postoperative management. Findings from this study demonstrated painful voiding in the first week was seen in $14 \%$ and $45 \%$ of stented and unstented patients, respectively. Additionally, none of the stented patients developed urinary retention or extravasation compared to $24 \%$ and $17 \%$ in unstented patients. In regards to postoperative meatal stricture, meatal dilatation was required in $6 \%$ of stented patients versus $17 \%$ of unstented patients. Finally, the re-operative rate trended lower in the urethral stented group ( $9 \%$ vs $20 \%$ ), though the difference was not statistically significant. ${ }^{25}$ Based on these findings, his conclusion was that urethral stenting after TIP repair for hypospadias is advantageous. 
Notwithstanding, several studies have evaluated healing in unstented hypospadias repair procedures. Hafez and colleagues utilized a rabbit model to study the temporal healing after TIP urethroplasty. They used a combination of retrograde urethrograms and microscopic examination at varying intervals and found no instances of fistulae or stricture in 13 performed procedures. Additionally, complete healing of the peri-urethral connective tissue with minimal fibrosis was seen. ${ }^{26}$ This has since been replicated numerous times in boys with several studies demonstrating that successful hypospadias repair is independent of the use of a urethral stent. ${ }^{27}$

Now the use of urethral stenting and urinary diversion after hypospadias repair rests mostly on surgeon preference and training, or in the case of re-do repairs. Most options for transurethral drainage include a modified catheter of adequate size, typically $6 \mathrm{Fr}$ or $8 \mathrm{Fr}$, or a feeding tube ranging 5-8 Fr placed atraumatically with verified drainage and secured via a glans stay suture (Figure 2). Time to removal varies too, with a traditional dwell time of 7 days, though recent studies have shown that removal postoperative day 1 (overnight urethral stenting), is not associated with any significant increase in adverse outcomes. ${ }^{28}$

\section{Urinary Retention}

De-novo urinary retention outside the setting of postoperative urinary retention, known neurological disorder and neonates is a rare entity. In their comprehensive review of pediatric presentation of acute urinary retention in the emergency setting between 2000 and 2012, Nevo et al found an overall incidence of urinary retention of 8.48 cases per 100,000 children. Additionally, $75 \%$ of children presenting were male. Underlying causes included mechanical obstruction (25\%), infection or inflammation (18\%), severe fecal constipation (13\%), and previously unknown neurologic disorders (11\%). Interestingly, up to $21 \%$ were idiopathic. Of note, all patients with mechanical obstruction were boys and 5 of 14 patients had a pelvic tumor. ${ }^{29}$

Age was also a factor - a bimodal distribution was noted with $29 \%$ of events occurring between 3 and 5 years of age, and $32 \%$ between the ages of 10 and 13 . Based on the alarming rate of concerning underlying pathology, acute onset urinary retention in a previously healthy child - excluding the postoperative period, known neurological disorder, and neonate age - should prompt swift and comprehensive evaluation.

In another study, adverse drug effects (particularly with antihistamines and neuroleptics such as haloperidol and carbamazepine) and behavioral dysfunctional voiding were six times more common in boys. ${ }^{13}$

\section{Neurogenic Bladder}

Neurogenic bladder or neurogenic detrusor-sphincter dysfunction encompasses abnormalities in the voiding arc that may develop due to a lesion at any level in the nervous system. This condition contributes to various forms of lower urinary tract dysfunction and can contribute to urinary tract infections, incontinence, and vesicoureteral reflux. In the long term, it can lead to renal scarring and compromised upper tracts, portending to chronic renal failure. ${ }^{30}$

The main goals of therapy are aimed to prevent deterioration of the urinary tract and to help the child achieve continence at an appropriate age. Management of neurogenic bladder, and its associated sequelae, has seen enormous gains, in large part to the introduction of clean

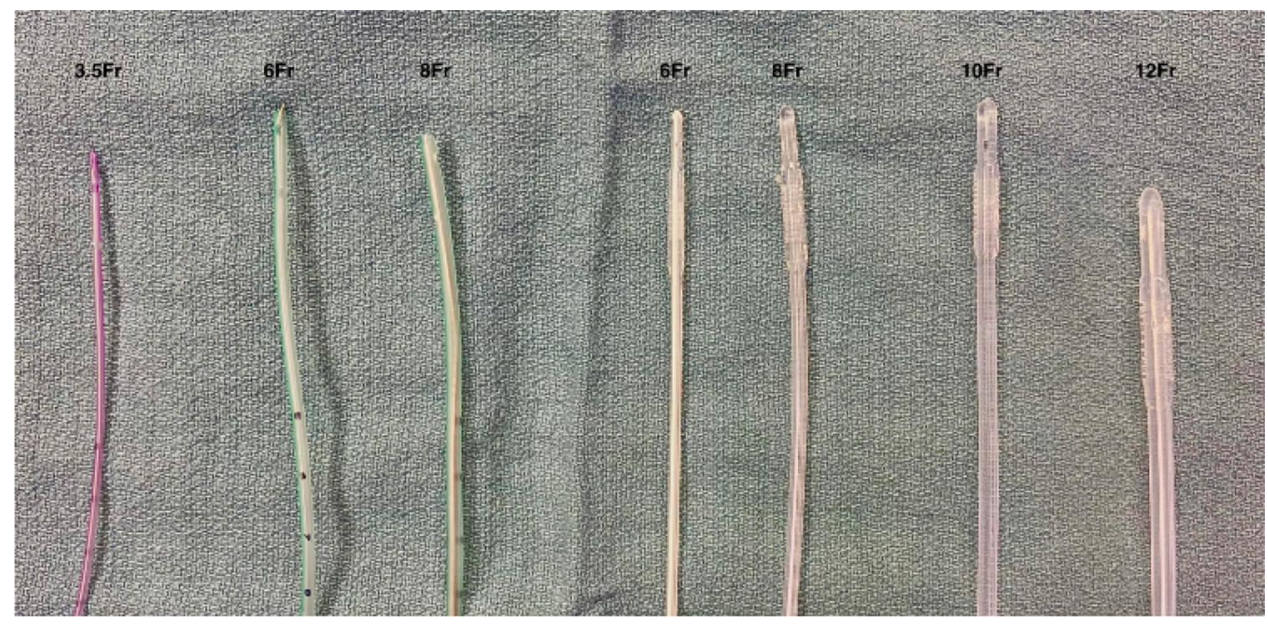

Figure 2 Urinary diversions - urethral stents and urinary catheters. Left half of picture represents feeding tubes which may be fashioned into urethral stents, ranging $3.5-8$ Fr. On the right half of the picture are various catheters, 6-12 Fr. 
intermittent catheterization (CIC). This has increased adherence to conservative management principles, which has allowed deferment of surgical intervention, if needed, until an age where success is most likely.

European Association of Urology (EAU) emphasizes and celebrates the overwhelming success CIC has had on early management of neurogenic bladder, and how, if started soon after birth, children do not develop upper tract deterioration. ${ }^{30}$ Kochakarn et al detailed the basis for early $\mathrm{CIC}$ and its effect by randomizing children with neurogenic bladder secondary to myelomeningocele to early intervention ( 1 year of age or less, $n=36$ ) or late intervention (CIC $>3$ years old, $\mathrm{n}=31$ ). Children who were started on $\mathrm{CIC}$ later had earlier renal deterioration and worse renal function (measured by increases in blood urea nitrogen and serum creatinine). Hydronephrosis was observed in 10 patients who received early intervention and 18 patients who were in the later treatment group. Patients in the later treatment group also had earlier and more severe hydronephrosis. Interestingly, in cases where surgery was performed, in either group, results of surgery were better in those who received early CIC, though surgery was required earlier in the late CIC group. Of note, no difference in urinary tract infection was seen between the two groups. ${ }^{31}$ A major barrier to the success of CIC, however, relies on complete support and acceptance by the patient, parents, and schools to ensure timely drainage. $^{32}$

In those requiring $\mathrm{CIC}$, hydrophilic, self-lubricating catheters are preferred and are associated with decreased risk of symptomatic urinary tract infection, bacteriuria, hematuria and pain, and increased patient satisfaction. ${ }^{33}$

For patients who have progressively worsening renal function despite CIC, definitive interventions include Mitrofanoff, Monti, or bladder augmentation. In such cases, a carefully selected, age-appropriate, urethral catheter is left indwelling for three to four weeks typically. For redundancy, a minimum of a $12 \mathrm{Fr}$ catheter is also placed suprapubically to maximize drainage and also irrigate mucus debris as needed. This is typically removed one to two weeks after the urethral catheter is removed and the patient is able to reliably demonstrate appropriate manipulation of the reconstructed diversion.

\section{Posterior Urethral Valves}

With an estimated incidence of 1 in 5000-12,500 livebirths, posterior urethral valves (PUV) are one of the few life-threatening congenital anomalies of the urinary tract. ${ }^{34,35}$ Often diagnosed antenatally on routine ultrasonography, proper management is imperative to avoid continued, and typically irreversible, kidney damage. Antenatal management is a rather recent treatment option afforded by incredible achievements in maternal-fetal management, technology, and bold surgical sense. A detailed discussion of such treatment is beyond the scope of this text, but antenatal treatment is reserved for only the most severe cases and consists of percutaneous vesicoamniotic shunt placement. ${ }^{36}$ This intervention is only offered at the most specialized of institutions that are able to offer jointly the obstetric, pediatric urologic, and potential pediatric care necessary to best maximize success.

Postnatally, if PUV is suspected in a newborn male, prompt drainage of the bladder is necessary, followed by voiding cystourethrogram (VCUG), if possible, which will demonstrate the classic "keyhole" sign (Figure 3). A neonate may be decompressed with a feeding tube ranging from 3.5 to $8 \mathrm{Fr}$, though $5 \mathrm{Fr}$ is typically performed. $6 \mathrm{Fr}$ balloon catheters may also be utilized, and in rarer instances, 4.8 or 6 Fr ureteral double j-coiled stents can be used. Of note, the utmost care must be taken to not

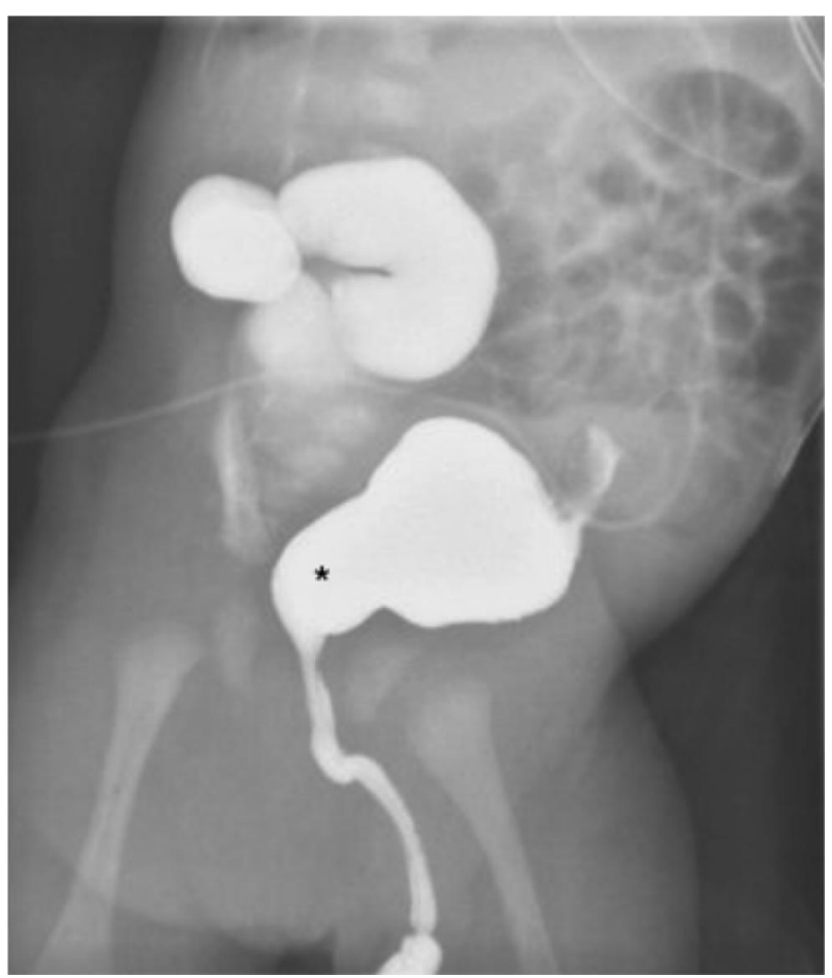

Figure 3 Voiding Cystourethrogram (VCUG) of male infant demonstrating dilated prostatic urethra with classic "key hole" sign secondary to posterior urethral valve. Some upper tract deterioration is already noted on the right given the significant dilatation from increased intravesical pressures. 
inflate the balloon within the urethra, inevitably causing more trauma. It is our recommendation that a $5 \mathrm{Fr}$ feeding tube or double-j stent over a guidewire be placed if possible and confirmed with bedside fluoroscopy or ultrasound. However, a 6 Fr urethral catheter may also be used without inflating the balloon and appropriately secured with tegaderm or tape. ${ }^{30}$ Alternative methods, if the transurethral approach fails, include suprapubic catheter insertion or cutaneous vesicostomy. Once the urinary system is decompressed, valve ablation typically is the definitive intervention and is performed once the child is safe to undergo anesthesia. A urethral catheter is placed during definitive treatment and left in place until the creatinine nadirs, which typically occurs 24 to 48 hours after repair.

\section{Urinary Decompression After Urologic Reconstruction}

The role of urinary drainage via trans-anastomotic ureteral stenting or percutaneous drainage remains heavily debated with the emergence of "stent-less" ureteral reconstruction, specifically in pyeloplasty. ${ }^{37}$ While this debate continues for upper tract urinary decompression, urethral catheterization is seemingly ubiquitous to drain the lower urinary tract. Conventionally, the bladder is decompressed for at least 24 hours, or overnight, in instances where extravesical ureteral reimplantation is utilized unless the patient has a history of voiding or bowel dysfunction, or both. More variability is seen in intravesical ureteral reimplant as the bladder wall and mucosa are violated. Urethral catheter dwell time in these instances is based on surgeon preference.

\section{Catheter Placement in the Pediatric Trauma Patient}

As previously mentioned, relative indications for immediate urethral catheter placement in the setting of pediatric trauma include unstable pelvic fractures, known trauma to the urethra, or blood at the meatus.

The bladder is the second most common genitourinary (GU) injury in children, owing to the proportionately larger aspect in children and lack of protection from the pubic symphysis due to incomplete lie within the pelvis from immature skeletal development. ${ }^{38,39}$ Bladder injuries can generally be categorized as either intraperitoneal or extraperitoneal. Cystography or computed tomography (CT) are preferred to plain film, with CT imaging the modality of choice in evaluating a suspected bladder injury. Prompt surgical exploration is required for intraperitoneal ruptures to avoid intraperitoneal infection, peritonitis and death. Adequate urethral catheter drainage by ensuring continuous drainage free of clots or debris - is typically sufficient for uncomplicated extraperitoneal rupture. Adequate drainage is ensured by appropriate size selection, as previously mentioned, and gentle irrigation with normal saline as needed to guarantee patency.

Rarely, urethral catheter drainage is also used in conjunction with ureteral stenting for upper tract (ureter and/ or renal) injuries to guarantee a low-pressure system with continued drainage in instances where urinary extravasation is noted from the proximal ureter or renal pelvis. ${ }^{39}$

Blood at the meatus in a presenting pediatric trauma patient warrants careful consideration. Current American Urological Association (AUA) guidelines on Urotrauma provide a stepwise approach for such a patient. Guideline statement 19 recommends clinicians should perform retrograde urethrography after pelvic or genital trauma resulting in blood being seen at the meatus. If partial disruption is noted with some contrast passing to the bladder, then the guidelines emphasize that a single attempt with a welllubricated catheter may be attempted by an experienced provider, in this case, a urologist. If complete distraction is noted, suprapubic tube placement is warranted; additionally, in most cases of pelvic fracture associated with urethral injury (PFUI), suprapubic drainage is preferred. ${ }^{40,41}$

\section{Catheter Preference in Female and Male Patients}

Cather preference is less an issue when considering gender difference than ensuring strict sterile technique. Aside from an obvious abnormality (such as hypospadias or stricture) the anatomy of a male infant is similar to that of an adult male, though smaller and lacking secondary sexual characteristics. A physiologic phimosis is frequently encountered during childhood, but the foreskin should not be forced to be retracted. Care should be taken to avoid forceful catheter placement as the tissue of a male infant is much less resilient compared to an adult male. ${ }^{18}$

Again, the most important factor when it comes to Foley placement in male versus female patients, is technique. In females, exposure is important and may require additional personnel to ensure contamination is minimized. This increased risk of infection in females results from their significantly shorter urethra $(4 \mathrm{~cm}$ in adult female vs $20 \mathrm{~cm}$ in adult male). Anatomic differences 


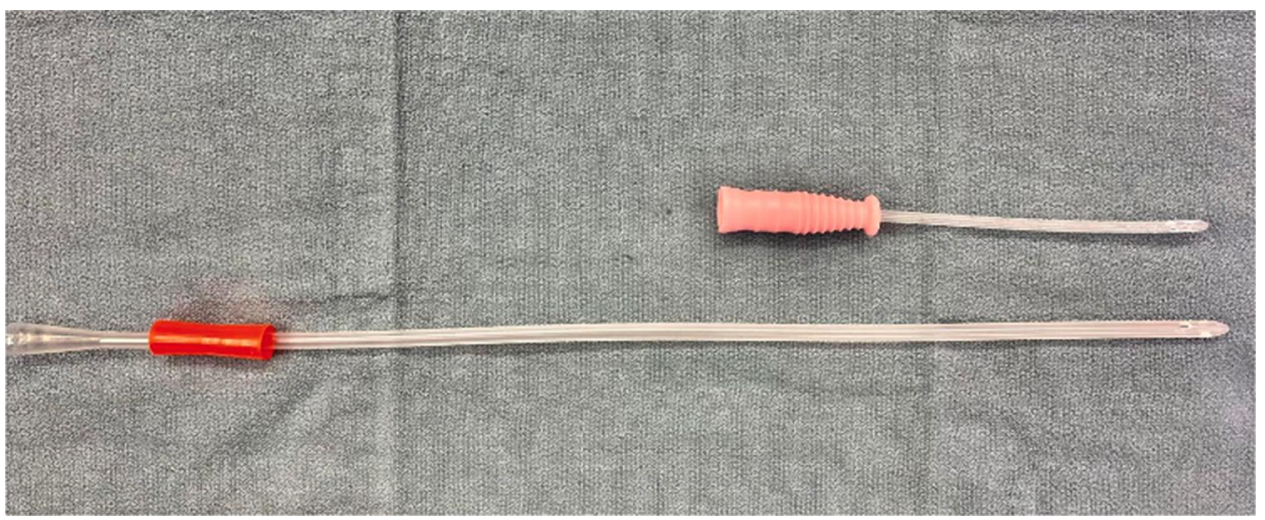

Figure 4 Urinary catheters for intermittent catheterization, 12 Fr female catheter (top) vs 12 Fr male catheter (bottom) representing anatomic difference in overall urethral length (Magic ${ }^{3} \mathrm{GO}$ Hydrophilic catheters, BARD).

are reflected in the difference in size of male and female catheters used in CIC (Figure 4).

Two common scenarios in male pediatric urologic patients cause great anxiety amongst guardians and providers unfamiliar with their presentation - uncorrected hypospadias and uncircumcised boys with physiologic phimosis. Anecdotally, the urethral opening and plate in uncorrected hypospadias often is able to accommodate normal, ageappropriate, catheterization in the absence of a concomitant urethral stricture, which is exceedingly rare in uncorrected hypospadias. In the setting of physiologic phimosis in the male infant, spatial awareness of the expected location of the meatus can often time allow passage of the urethral catheter. Another, well-documented approach, is the application of steroid cream with gentle retraction to manual release any adhesions. If the acuity necessitates more immediate attention, dorsal slit may always be performed. Knowledge of these common conditions, and how to address each, can greatly reduce many phone calls the pediatric provider experiences.

\section{Role of Antibiotics and Catheter Materials \\ Antibiotic Prophylaxis - Is There a Role?}

Much like the role of urethral stenting in hypospadias repair, there is substantial variation in practice methods amongst pediatric urologists with regards to antibiotic prophylaxis with urinary catheterization and minor lower urinary tract procedures. Although guidelines exist, these are geared toward the adult urologic patient.

To address this chasm, in 2017, Glaser and associates surveyed members of the Society for Pediatric Urology regarding antibiotic use with catheterization. A significant majority, 78\%, prescribed daily prophylaxis with a hypospadias stent in place, but disagreement abound thereafter. Interestingly, urologists over 50 years and fellowship-trained pediatric urologists were more likely to prescribe prophylaxis for hypospadias stents. Extensive variation existed with prophylaxis for Foley catheters, percutaneous nephrostomy tubes, suprapubic tubes and internal double- $\mathrm{j}$ ureteral stents, with $30-50 \%$ of respondents not prescribing prophylaxis for these drainage systems. Additionally, the majority of respondents do not routinely prescribe a dose of prophylaxis prior to tube removal aside from removal of a ureteral stent. ${ }^{42}$

The use of urine cultures and culture data differed widely, too. The majority do not routinely obtain urine culture prior to removal of a hypospadias stent (90\%), Foley catheter (75\%), nephrostomy tube (59\%), suprapubic tube $(69 \%)$ or ureteral stent (67\%). A void for consensus regarding prophylaxis in the pediatric-patient exists with such wide variation in actual practice, coupled with the potential cost of treatment and morbidity for urinary tract infection. This topic remains heavily debated, and researched.

In 2018 Canon and colleagues shed light on this debate in their evaluation of the incidence of symptomatic urinary tract infection (UTI) following stented, distal hypospadias repair. Additionally, they evaluated the impact of prophylactic antibiotic therapy on UTI incidence. Urinalysis and/or urine cultures were obtained intraoperatively from 48 patients aged 0 to 5 years who did not receive preoperative antibiotic regimens. These patients were randomized to prophylaxis vs no prophylaxis groups in equal numbers. While the incidence of symptomatic UTI was low in this small pilot study, it did not vary between groups and 
prophylactic antibiotic therapy did not appear to lower the incidence of symptomatic UTI. ${ }^{43}$

\section{Catheter Coatings}

Biofilm formation and encrustation are two dreaded natural progressions with repeated and chronic catheter use. Both can develop despite appropriate oral or intravenous antimicrobial therapy. With the increased morbidity associated, many have considered special catheter materials to reduce formation. Johnson and others sought to answer how coated catheters faired against traditional silicone or latex foleys. In a meta-analysis totaling over 13,000 patients, they found there was low-quality evidence favoring nitrofurazone-impregnated catheters in patients catheterized for short durations, less than one week. The benefit was observed in an overall decreased risk of bacteriuria without any increased risk of antimicrobial resistance. ${ }^{44}$

Other catheter coatings have also shown some modest benefit. Silver-coated catheters have been heavily studied and low-quality evidence suggests a benefit over traditional latex catheters. Endpoints demonstrated a decreased risk of bacteriuria and no evidence of urethral irritation or antimicrobial resistance. Differences were significant only with silver alloy-coated catheters but not silver oxide-coated catheters. Specifically, silver alloy-coated catheters reduced the risk of asymptomatic bacteriuria compared to standard latex catheters, but this significance was lost when compared to standard, all-silicone catheters. ${ }^{45}$

Traditional latex catheters have fallen out of favor due to concerns over latex allergy. Silicone is a ubiquitous catheter material now and is preferred in patients with long-term catheterization due to decreased likelihood of catheter encrustation. For those patients prone to catheter blockage secondary to encrustation, silicone catheters should be utilized.

\section{Catheter Dwell Time}

Just as significant consideration afforded to determining appropriateness of urinary catheter placement, equal consideration should be given to urinary catheter dwell time. To assess the risk of catheter dwell time, Letica-Kriegel et al studied a large retrospective cohort of 61,047 patients from six different hospitals to assess how the risk for CAUTI changes over time. Their results demonstrated that each additional day of catheterization increases the risk of CAUTI, even when controlling for sex, age, and patient comorbidities. Additionally, they also identified female sex, pediatric age, and underlying neurologic issues as independent risk factors for CAUTIs. ${ }^{46}$ These findings confirmed the long-held practice and drive amongst surgical teams to remove urinary catheters as soon as possible (when safely and feasible). It also served as a modern update to the classic finding that adult patients with urinary catheters develop bacteriuria at a rate of $8 \%$ per day during the first week. ${ }^{47}$

\section{Conclusion}

In the larger context of perioperative management, urinary catheter selection in the pediatric is often an afterthought. In cases where urinary diversion is utilized, existing knowledge regarding catheter selection often complicates the process. A step-wise process should be employed and begins with determining the need for catheter placement in the first place.

If warranted, either due to anticipated operative time, immobility, or pelvic laparoscopic surgery, amongst other reasons, careful attention to technique must be emphasized. The correct size for the pediatric patient, based on height and weight, aids in proper catheterization. Silicone-based catheters predominate currently and should be utilized when available. Once placed, a push should be made to remove the catheter as soon as medically necessary, with a keen focus on antibiotic prophylaxis only if necessary, to maintain antimicrobial stewardship. In cases where postoperative urinary retention, or underlying disease states (such as neurogenic bladder) may predispose to recatheterization, CIC should always be employed first until no longer feasible- due to trauma or patient condition. Taken together, these mental checkpoints can ensure catheter usage remains judicious to decrease the use of CAUTI and any subsequent sequelae in the pediatric surgical patient.

\section{Disclosure}

The authors reported no conflicts of interest for this work.

\section{References}

1. Tzong KY, Han S, Roh A, Ing C. Epidemiology of pediatric surgical admissions in US children: data from the HCUP kids inpatient database. J Neurosurg Anesthesiol. 2012;24(4):391-395. PMID: 23076227. doi:10.1097/ANA.0b013e31826a0345

2. Munnich EL, Parente ST. Procedures take less time at ambulatory surgery centers, keeping costs down and ability to meet demand up. Health Aff. 2014;33(5):764-769. doi:10.1377/hlthaff.2013.1281

3. Klevens RM, Edwards JR, Richards CL, et al. Estimating health care-associated infections and deaths in U.S. hospitals, 2002. Public Health Rep. 2007;122(2):160-166. doi:10.1177/003335490712200205 
4. Hidron AI, Edwards JR, Patel J, et al. Antimicrobial-resistant pathogens associated with healthcare-associated infections: annual summary of data reported to the national healthcare safety network at the centers for disease control and prevention, 2006-2007. Infect Control Hosp Epidemiol. 2008;29(11):996-1011. doi:10.1086/591861

5. Cho HJ, Khalil S, Poeran J, et al. Lose the tube: a choosing wisely initiative to reduce catheter-associated urinary tract infections in hospitalist-led inpatient units. Am J Infect Control. 2017;45 (3):333-335. doi:10.1016/j.ajic.2016.10.023

6. Meddings J, Skolarus TA, Fowler KE, et al. Michigan Appropriate Perioperative (MAP) criteria for urinary catheter use in common general and orthopaedic surgeries: results obtained using the RAND/UCLA appropriateness method. BMJ Qual Saf. 2018;28 (1):56-66. doi:10.1136/bmjqs-2018-008025

7. Tammela T, Kontturi M, Lukkarinen O. Postoperative urinary retention: I. Incidence and predisposing factors. Scand J Urol Nephrol. 1986;20(3):197-201. doi:10.3109/00365598609024494

8. Keskinen H, Helenius L, Pajulo O, Helenius IJ. Postoperative urinary retention or difficulties to empty the bladder in young patients undergoing posterior spinal fusion for adolescent idiopathic scoliosis. $J$ Pediatr Surg. 2018;53(8):1542-1546. PMID: 29153469. doi:10.1016/j.jpedsurg.2017.09.023

9. Agrawal K, Majhi S, Garg R. Post-operative urinary retention: review of literature. World J Anesthesiol. 2019;8(1):1-12. doi:10.5313/wja.v8.i1.1

10. Baldini G, Bagry H, Aprikian A, Carli F, Warner DS, Warner MA. Postoperative urinary retention. Anesthesiology. 2009;110 (5):1139-1157. PMID: 19352147. doi:10.1097/aln.0b013e31819f7aea

11. Cropper J, Hutchison L, Llewellyn N. Post-operative retention of urine in children. Paediatr Nurs. 2003;15(7):15-18. doi:10.7748/ paed.15.7.15.s21

12. Kemp D, Tabaka N. Postoperative urinary retention: part II-A retrospective study. J Post Anesth Nurs. 1990;5(6):397-400.

13. Gatti JM, Perez-Brayfield M, Kirsch AJ, Smith EA, Massad HC, Broecker BH. Acute urinary retention in children. J Urol. 2001;165 (3):918-921. PMID: 11176514. doi:10.1016/S0022-5347(05)66574-3

14. Pavlin DJ, Pavlin EG, Gunn HC, Taraday JK, Koerschgen ME. Voiding in patients managed with or without ultrasound monitoring of bladder volume after outpatient surgery. Anesth Analg. 1999;89 (1):90-97. doi:10.1097/00000539-199907000-00016

15. Rosseland LA, Stubhaug A, Breivik H. Detecting postoperative urinary retention with an ultrasound scanner. Acta Anaesthesiol Scand. 2002;46(3):279-282. PMID: 11939918. doi:10.1034/j.13996576.2002.t01-1-460309.x

16. Keita H, Diouf E, Tubach F, et al. Predictive factors of early postoperative urinary retention in the postanesthesia care unit. Anesth Analg. 2005;101 (2):592-596. doi:10.1213/01.ane.0000159165.90094.40

17. Shilkofski N; Procedures. Body fluid sampling. E. Urinary bladder catheterization. In: Robertson J, Shilkofski N, editors. The Harriet Lane Handbook. 17th ed. Philadelphia: Elsevier Mosby; 2005:94.

18. Lacroix LE, Vunda A, Bajwa NM, Galetto-Lacour A, Gervaix A. Catheterization of the urethra in male children. $N$ Engl $\mathrm{J} \mathrm{Med}$. 2010;363(14):14. doi:10.1056/nejmvem0808873

19. Custer JW, Rau RE. Pediatric parameters and equipment. In: Custer JW, Rau RE, editors. The Harriet Lane Handbook. 18th ed. Philadelphia: Elsevier Mosby; 2009.

20. Chapter 12. Pediatric trauma. In: Hazinski MF, editor. Manual of Pediatric Critical Care. 1st ed. St. Louis: Mosby-Year Book; 1999:590.

21. Broselow pediatric resuscitation tape (adapted from). Lincolshire, IL: Armstrong Medical Industries; 2011. Product No: AE-4800.

22. Biarent D, Bingham R, Rodriguez-Nuňez A, et al. European PaeDiatric Life Support Course Manual. 2nd ed. Antwerp-Belgium: EuropeanResuscitation Council (ERC); 2003:7-9.

23. Kopač M. Formula estimation of appropriate urinary catheter size in children. $J$ Pediatr Intensive Care. 2015;02(04):177-180. doi:10.3233/pic-13069
24. Snodgrass WT. Snodgrass technique for hypospadias repair. BJU Int. 2005;95(4):683-693. doi:10.1111/j.1464-410x.2005.05384.x

25. El-Sherbiny M. Tubularized incised plate repair of distal hypospadias in toilet-trained children: should a stent be left? BJU Int. 2003;92 (9):1003-1005. PMID: 14632864. doi:10.1111/j.1464-410x.20 03.04513.x

26. Hafez A, Herz D, Bägli D, Smith C, Mclorie G, Khoury A. Healing of unstented tubularized incised plate urethroplasty: an experimental study in a rabbit model. BJU Int. 2003;91(1):84-88. doi:10.1046/ j.1464-410x.2003.03084.x

27. Hakim S, Merguerian PA, Rabinowitz R, Shortliffe LD, McKenna PH. Outcome analysis of the modified Mathieu hypospadias repair: comparison of stented and unstented repairs. $J$ Urol. 1996;156(2S):836-838. doi:10.1016/s0022-5347(01) 65834-8

28. Ritch CR, Murphy AM, Woldu SL, Reiley EA, Hensle TW. Overnight urethral stenting after tubularized incised plate urethroplasty for distal hypospadias. Pediatr Surg Int. 2010;26(6):639-642. doi:10.1007/s00383-010-2605-6

29. Nevo A, Mano R, Livne PM, Sivan B, Ben-Meir D. Urinary retention in children. Urology. 2014;84(6):1475-1479. PMID: 25440826. doi:10.1016/j.urology.2014.08.020

30. Radmayr C, Bogaert G, Dogan HS, et al. EAU guidelines: paediatric urology. Uroweb; 2018. Available from: https://uroweb.org/wpcontent/uploads/EAU-Guidelines-on-Paediatric-Urology-2018-largetext.pdf. Accessed November 21, 2020.

31. Kochakarn W, Ratana-Olarn K, Lertsitchichai P, Roongreungsilp U. Follow-up of long-term treatment with clean intermittent catheterization for neurogenic bladder in children. Asian J. 2004;27 (2):134-136.

32. Cass A, Luxenberg M, Gleich P, Johnson C, Hagen S. Clean intermittent catheterization in the management of the neurogenic bladder in children. $J$ Urol. 1984;132(3):526-528. PMID: 6471189. doi:10.1016/s0022-5347(17)49720-5

33. Cardenas DD, Moore KN, Dannels-McClure A, et al. Intermittent catheterization with a hydrophilic-coated catheter delays urinary tract infections in acute spinal cord injury: a prospective, randomized, multicenter trial. PMR. 2011;3(5):408-417. doi:10.1016/j. pmrj.2011.01.001

34. Smith GHH, Canning DA, Schulman SL, Snyder HM, Duckett JW. The long-term outcome of posterior urethral valves treated with primary valve ablation and observation. $J$ Urol. 1996;155 (5):1730-1734. doi:10.1097/00005392-199605000-00065

35. Atwell J. Posterior urethral valves in the British Isles: a multicenter B.A.P.S. review. J Urol. 1983;130(4):835-836. doi:10.1016/s00225347(17)51505-0

36. Fishberg SE, Landau EH, Duvdevani M, Gofrit ON, Friedman SE, Hidas G. Posterior urethral valves: prenatal, neonatal, and long-term management. American Academy of Pediatrics; December 1, 2018. Available from: https://neoreviews.aappublications.org/content/19/ 12/e753. Accessed January 11, 2021.

37. Silva MV, Levy AC, Finkelstein JB, Van Batavia JP, Casale P. Is peri-operative urethral catheter drainage enough? The case for stentless pediatric robotic pyeloplasty. J Pediatr Urol. 2015;11(4):175. e1-5. doi:10.1016/j.jpurol.2015.06.003

38. Mcaleer IM, Kaplan GW, Scherz HC, Packer MG, P.Lynch F. Genitourinary trauma in the pediatric patient. Urology. 1993;42 (5):563-567. doi:10.1016/0090-4295(93)90274-e

39. Deibert CM, Spencer BA. The association between operative repair of bladder injury and improved survival: results from the national trauma data bank. J Urol. 2011;186(1):151-155. doi:10.1016/j. juro.2011.03.002

40. Gaines B, Austin K. Chapter 16: Abdominal and Renal Trauma. In: Ashcraft's Pediatric Surgery, 6th Edition. London: Elsevier Saunders; 2014:215-229. 
41. Morey AF, Brandes S, Dugi DD, et al. Urotrauma: AUA guideline. J Urol. 2014;192(2):327. doi:10.1016/j.juro.2014.05.004

42. Glaser AP, Rosoklija I, Johnson EK, Yerkes EB. Prophylactic antibiotic use in pediatric patients undergoing urinary tract catheterization: a survey of members of the Society for Pediatric Urology. $B M C$ Urol. 2017;17(1). doi:10.1186/s12894-017-0268-5

43. Canon S, Marquette MK, Crane A, Patel A, Zamilpa I, Bai S. Prophylactic antibiotics after stented, distal hypospadias repair: randomized pilot study. Glob Pediatr Health. 2018;5. doi:10.1177/ 2333794X18770074

44. Johnson JR, Kuskowski MA, Wilt TJ. Systematic review: antimicrobial urinary catheters to prevent catheter-associated urinary tract infection in hospitalized patients. Ann Intern Med. 2006;144(2):116. doi:10.7326/0003-4819-144-2-200601170-00009
45. Newton T, Still JM, Law EA. Comparison of the effect of early insertion of standard latex and silver-impregnated latex Foley catheters on urinary tract infections in burn patients. Infect Control Hosp Epidemiol. 2002;23(4):217-218. doi:10.1086/502038

46. Letica-Kriegel AS, Salmasian H, Vawdrey DK, et al. Identifying the risk factors for catheter-associated urinary tract infections: a large cross-sectional study of six hospitals. BMJ Open. 2019;9(2):e022137. PMID: 30796114; PMCID: PMC6398917. doi:10.1136/bmjopen2018-022137

47. Garibaldi RA, Burke JP, Dickman ML, Smith CB. Factors predisposing to bacteriuria during indwelling urethral catheterization. $N$ Engl J Med. 1974;291(5):215-219. doi:10.1056/nejm197408012910501

\section{Publish your work in this journal}

Research and Reports in Urology is an international, peer-reviewed, open access journal publishing original research, reports, editorials, reviews and commentaries on all aspects of adult and pediatric urology in the clinic and laboratory including the following topics: Pathology, pathophysiology of urological disease; Investigation and treatment of urological disease; Pharmacology of drugs used for the treatment of urological disease. The manuscript management system is completely online and includes a very quick and fair peer-review system, which is all easy to use. Visit http://www.dovepress.com/ testimonials.php to read real quotes from published authors. 